\title{
Epidemiological Aspects of Diabetic Retinopathy- A Narrative Review
}

\author{
Seyed Ahmad Rasoulinejad ${ }^{1}$ \\ ${ }^{1}$ Associate Professor, Department of Ophthalmology, School of Medicine, Social Determinants of Health Research \\ Center, Ayatollah Rouhani Hospital, Babol University of Medical Sciences, Babol, Iran.
}

\section{ABSTRACT}

Diabetic retinopathy is still the major cause of acquired loss of vision in the world among the middle-aged and thus economically active population. Understating epidemiology of diabetic retinopathy is helpful for conceptualization and progression of leading public health strategies such as screening programs for the disease. In the present review, we evaluated the data on incidence or development of diabetic retinopathy worldwide. This study was conducted by searching the scientific databases using the related keywords. Assessing the related papers showed that few studies have been conducted on the prevalence and incidence of diabetic retinopathy, however, the data revealed a progression in its epidemiology since last three decades. The annual incidence of diabetic retinopathy ranged from $2 \%$ to $13 \%$ in the world. An increase in age-standardized rate of blindness and vision impairment caused by diabetic retinopathy was seen in North Africa and the Middle East, while the prevalence of blindness and vision impairment due to diabetic retinopathy decreased in all areas of sub-Saharan Africa, Latin America, Oceania, Caribbean, as well as central and eastern Europe. Factors such as hyperglycaemia, hypertension, and dyslipidaemia have been shown to be positively associated with diabetic retinopathy; however, more studies are needed for confirming the relations. Although the global prevalence of diabetes mellitus is raising, no sufficient evidence exists regarding diabetic retinopathy epidemiology in most societies.
Corresponding Author: Dr. Seyed Ahmad Rasoulinejad, Department of Ophthalmology, School of Medicine,

Social Determinants of Health Research Center, Ayotollah Rouhani Hospital, Babol University of Medical Sciences, Babol, Mazandaran, Iran,

E-mail: sarasoulinejad123@gmail.com

DOI: $10.14260 / \mathrm{jemds} / 2019 / 708$

Financial or Other Competing Interests: None.

How to Cite This Article:

Rasoulinejad SA. Epidemiological aspects of diabetic retinopathy- a narrative review. J. Evolution Med. Dent. Sci. 2019;8(43): 3268-3272, DOI:
Submission 18-08-2019,

Peer Review 10-10-2019,

Acceptance 16-10-2019,

Published 28-10-2019. 


\section{BACKGROUND}

One of the fast-growing chronic diseases in the world is diabetes mellitus. The World Health Organization estimates that the overall number of people with diabetes will increase from 171 million people in 2000 to 366 million people by 2030.(1) Another report stated that diabetes had an effect on about 415 million people in the world in 2015, and this estimate is expected to increase to 642 million people by 2040.(2) Diabetes mellitus is a major cause of avoidable blindness in working age population globally. Diabetic retinopathy, which is a specific microvascular complication of diabetes mellitus, is still the major cause of acquired loss of vision in the world among the middle-aged and thus economically active population.(3) The potential risk of blindness in a person with diabetes is nearly three times greater than that of a healthy person.(4) As the prevalence of diabetes and the number of people with diabetes who live longer is rising, the number of people with diabetic retinopathy and visual impairment due to this disease is increasing in the world.(5) Diabetic retinopathy may not have any symptoms in the early stages. However, symptoms that could indicate that the disease has progressed to a more advanced stage include blurred vision, spots that float in the vision, halos around lights, loss of central vision, and loss of color vision. $(6,7)$ This review article aims to explore the epidemiological aspects of diabetic retinopathy in the world, in order to implement appropriate strategies to screen and manage the disease.

\section{Search Strategy and Results}

This study was conducted using the keywords of "diabetic retinopathy", "epidemiology", "prevalence" and "incidence" through searching in the scientific databases of PubMed, Scopus and Web of Science. Unrelated sources and articles were excluded. Assessing the related papers showed that despite the momentousness of diabetic retinopathy and the increasing prevalence of diabetes particularly in Asian countries like India and China, ${ }^{(8)}$ few studies have focused on the incidence of this disorder around the world, especially severe sight-threatening stages of the disease, including proliferative diabetic retinopathy and diabetic macular oedema. With the increase in the number of people who suffer from diabetes, the number of diabetic retinopathy and vision-threatening diabetic retinopathy, which involves severe non-proliferative diabetic retinopathy, proliferative diabetic retinopathy and diabetic macular oedema, is estimated to increase to 191 million people and 56.3 million people, respectively by $2030 .{ }^{(9)}$

Previous individual studies have shown considerable discrepancy in the estimates for the prevalence of diabetic retinopathy among people with both diagnosed and undiagnosed diabetes. Of course, differences in study methodologies, population features, and ascertainment and classification of diabetic retinopathy have made it difficult to compare the studies directly. A recent meta-analysis stated that the annual incidence of diabetic retinopathy ranged from $2.2 \%$ to $12.7 \%{ }^{(10)}$ The crude prevalence (All ages) of vision impairment and blindness caused by diabetic retinopathy increased significantly from 1990 to 2015.(11) The number of patients that were influenced by blindness due to diabetic retinopathy raised from 0.2 million to 0.4 million, and moderate to severe vision impairment increased from 1.4 million to 2.6 million. An increase in age-standardized rate of blindness and vision impairment caused by diabetic retinopathy was seen in North Africa and the Middle East, while the prevalence of blindness and vision impairment due to diabetic retinopathy decreased in all areas of sub-Saharan Africa, Latin America, Oceania, Caribbean, as well as central and eastern Europe.(11) In the following, we presented reports on epidemiology of diabetic retinopathy around the world.

\section{United States}

From 2010 to 2050, the number of Americans with diabetic retinopathy is expected to nearly double, from 7.7 million to 14.6 million.(12) A meta-analysis on eight population-based eye surveys summarized the prevalence of diabetic retinopathy in the United States.(13) The authors reported that the pooled prevalence rates for retinopathy and visionthreatening retinopathy were $40.3 \%$ and $8.2 \%$ among 10.2 million adults aged $\geq 40$ years old with diabetes mellitus, respectively. These rates among general population were $3.4 \%$ and $0.75 \% .{ }^{(13)}$ A recent study on about one thousand subjects with diabetes showed that $25.4 \%$ of the patients had mild and moderate non-proliferative diabetic retinopathy, and $2.3 \%$ had severe non-proliferative diabetic retinopathy or proliferative diabetic retinopathy.(14) Another investigation in the United States indicated that out of 2240 youths with type 1 diabetes mellitus and 1768 youths with type 2 diabetes mellitus, $20.1 \%$ and $7.2 \%$ developed diabetic retinopathy over a median follow-up time of 3.2 and 3.1 years, respectively, showing that youths with types 1 or 2 diabetes mellitus are at a considerable risk for diabetic retinopathy and should undergo regular screenings.(15)

\section{Europe}

In the United Kingdom, data of the Clinical Practice Research Datalink electronic health database on 338,390 patients with type 2 diabetes and 30,657 patients with type 1 diabetes indicated that the prevalence of diabetic retinopathy was $48.4 \%$ in patients with type 1 diabetes mellitus and $28.3 \%$ in patients with type 2 diabetes mellitus.(16) This rate in Portugal, based on the Diabetic Retinopathy Screening Service for Lisbon and Tagus Valley (RETINODIAB) between 2009 and 2014 on 52,739 people with type 2 diabetes, was 16.3\%. Among these patients, $10.4 \%$ had mild nonproliferative diabetic retinopathy, $2.8 \%$ had moderate nonproliferative diabetic retinopathy, $1.3 \%$ had severe nonproliferative diabetic retinopathy and $1.8 \%$ had proliferative diabetic retinopathy. Statistical analysis demonstrated that the presence of any diabetic retinopathy, non-referable diabetic retinopathy or referable diabetic retinopathy was highly related to increased duration of diabetes and earlier age at diagnosis.(17) In Germany and Austria, a survey conducted during 2000-2013 on the German/Austrian Diabetes Prospective Documentation Initiative data ${ }^{7}$, on 64,784 patients with type 2 diabetes, exhibited that $10.26 \%$ of patients had mild to moderate retinopathy and $9.09 \%$ of patients had severe non-proliferative or proliferative retinopathy. Additionally, $0.77 \%$ had diabetic macular oedema.(18) From Denmark, the Danish Registry of Diabetic Retinopathy data on 77,968 patients with diabetes aged more than 18 years showed that the prevalence of non-sight- 
threatening diabetic retinopathy, non-proliferative diabetic retinopathy and proliferative diabetic retinopathy was $78 \%$, $18 \%$ and $4 \%$ in the study population, respectively. Nevertheless, the total prevalence of patients without diabetic maculopathy was $97 \%$ in this registry.(19) In a population-based prospective study from Spain on 15,396 patients with diabetes mellitus, an increase in the incidence of diabetic retinopathy and diabetic macular oedema could be observed, particularly in patients who were 31 to 70 years old, and it was associated with poor metabolic control of diabetes mellitus. The incidence of any type of diabetic retinopathy raised from $8.09 \%$ in 2007 to $8.99 \%$ in 2014 , and diabetic macular oedema raised from $2.00 \%$ in 2007 to $2.49 \%$ in 2014.(20)

\section{Asia}

A recently published meta-analysis in Iran on thirty-one studies including 23,729 patients with types I and II diabetes revealed that prevalence of diabetic retinopathy, nonproliferative diabetic retinopathy and proliferative diabetic retinopathy were $41.9 \%, 32.2 \%$, and $13.2 \%$, respectively. (21) From China, a current study used data gathered from eight hospitals between 2014 and 2016 on 16,305 patients with diabetes mellitus. The age-standardized prevalence rates of any diabetic retinopathy and sight-threatening diabetic retinopathy were $27.9 \%$ and $12.6 \%$, respectively. It was demonstrated that younger age and lower triglycerides had a protective role against diabetic retinopathy, and longer duration of diabetes, higher haemoglobin A1c levels, higher fasting plasma glucose, higher systolic blood pressure, faster heart rate, higher low-density lipoprotein, higher blood urea nitrogen and elevated serum creatinine level were positively related to the presence of any diabetic retinopathy. Similar risk factors, except for blood urea nitrogen and triglycerides, were reported for sight-threatening diabetic retinopathy.(22) A study from Singapore on the Singapore Epidemiology of Eye Diseases Study data, which was performed from 2004 until 2011, 2,376 patients suffered from diabetes mellitus, $33.9 \%$ of them had diabetic retinopathy and $26.3 \%$ had vision-threatening diabetic retinopathy. There was not any significant difference in age-standardized prevalence of diabetic retinopathy and vision-threatening diabetic retinopathy between different ethnic groups. Among patients with diabetic retinopathy, $81.6 \%$ had minimal to moderate non-referable diabetic retinopathy, $18.4 \%$ had severe nonreferable diabetic retinopathy or proliferative diabetic retinopathy and $12.8 \%$ had clinically significant macular oedema.(23) A recent study from India showed an incidence rate of $2.4 \%$ per year for diabetic retinopathy. ${ }^{(24)}$

\section{Africa}

A population-based cohort study in Kenya, sub-Saharan Africa, by Bastawrous et al.(25) demonstrated that annual incidence of diabetic retinopathy was 224.7 per 1000 among patients with known diabetes mellitus at baseline. Diabetic retinopathy was strongly associated with increasing age, higher body mass index, urban dwelling and higher socioeconomic status.

\section{Oceania}

In the Greater Wellington area of New Zealand, data related to 12,667 patients who referred from 2006 until 2015 for their first diabetic retinal screening were assessed. The prevalence of any diabetic retinopathy was $22.5 \%$, consisting of $20.2 \%$ with non-sight-threatening diabetic retinopathy and $2.3 \%$ with sight-threatening diabetic retinopathy. It was also showed that type 1 diabetes and poor haemoglobin A1c control were highly related to any degree of diabetic retinopathy. Old age ( $>65$ years) increased the risk of the disease, while Asian and Pacific Island ethnicity had relatively higher odds compared to European ethnicity.(26)

\section{DISCUSSION}

Although the global prevalence of diabetes mellitus is raising, no sufficient evidence exists on the diabetic retinopathy epidemiology in most societies. Interpreting the present incidence data from the mentioned diverse studies is not easy considering the extensive variation in estimates of different countries and regions, and due to differences in study methods, type of diabetes, and time indices. A previous systematic review that evaluated the development of diabetic retinopathy to proliferative diabetic retinopathy and severe sight loss demonstrated a declining development since 1980 in high-income countries.(27) Nevertheless, little data is available regarding the incidence and development of this disorder. Epidemiological data are of great importance for the expansion of crucial public health strategies such as screening programs.

It is commonly accepted that screening, early diagnosis and prompt treatment of vision-threatening forms of diabetic retinopathy can reduce the number of patients with diabetesrelated vision impairment.(28) Nevertheless, screening services for diabetic retinopathy in both developed and developing countries remain inconsistent, and are persistently faced with ambiguous guidelines about the most appropriate method for screening (e.g., clinical assessment vs fundus photography), while more resources are required for implementation and maintenance of a comprehensive screening program for diabetic retinopathy.(29) Thus, diabetic retinopathy is considered as an increasingly noteworthy public health problem, particularly in several middle-income and low-income countries in which access to trained eye-care professionals and secondary and tertiary eye-care services (e.g., access to laser and intravitreal treatment) may be substandard. Thus, it is essential that all public and private stakeholders search for novel ways to control diabetes mellitus, improve access to diabetic retinopathy screening, and plan and improve cost-effective screening programs in the society.

\section{CONCLUSIONS}

Although factors such as hyperglycaemia, hypertension, and dyslipidaemia have been shown to be positively associated with diabetic retinopathy.(30-32) more research is needed to support and confirm them as risk factors, especially with 
regard to severe stages of diabetic retinopathy. Finding a broader and more precise estimate of the prevalence of diabetic retinopathy and its relationship with the main adjustable risk factors, particularly for vision-threatening diabetic retinopathy, is essential for directing public health training and optimum clinical management of diabetes.

\section{REFERENCES}

[1] Wild S, Roglic G, Green A, et al. Global prevalence of diabetes: estimates for the year 2000 and projections for 2030. Diabetes Care 2004;27(5):1047-53.

[2] Ogurtsova K, da Rocha FJD, Huang Y, et al. IDF Diabetes Atlas: Global estimates for the prevalence of diabetes for 2015 and 2040. Diabetes Res Clin Pract 2017;128:40-50.

[3] Wang W, Lo ACY. Diabetic retinopathy: pathophysiology and treatments. Int J Mol Sci 2018;19(6). pii: E1816.

[4] Kyari F, Tafida A, Sivasubramaniam S, et al. Prevalence and risk factors for diabetes and diabetic retinopathy: results from the Nigeria national blindness and visual impairment survey. BMC Public Health 2014;14:1299.

[5] Zheng Y, He M, Congdon N. The worldwide epidemic of diabetic retinopathy. Indian J Ophthalmol 2012;60(5):428-31.

[6] Candrilli SD, Davis KL, Kan HJ, et al. Prevalence and the associated burden of illness of symptoms of diabetic peripheral neuropathy and diabetic retinopathy. J Diabetes Complications 2007;21(5):306-14.

[7] Mikaniki E, Rasoulinejad SA, GharehDaghly L, et al. The relationship between serum lipids and severity of macular oedema in diabetic patients. J Mazand Univ Med Sci 2011;20(80):8-15.

[8] Yau JW, Rogers SL, Kawasaki R, et al. Global prevalence and major risk factors of diabetic retinopathy. Diabetes Care 2012;35(3):556-64.

[9] Ting DS, Cheung GC, Wong TY. Diabetic retinopathy: global prevalence, major risk factors, screening practices and public health challenges: a review. Clin Exp Ophthalmol 2016;44(4):260-77.

[10] Sabanayagam C, Banu R, Chee ML, et al. Incidence and progression of diabetic retinopathy: a systematic review. Lancet Diabetes Endocrinol 2019;7(2):140-9.

[11] Flaxman SR, Bourne RRA, Resnikoff S, et al. Global causes of blindness and distance vision impairment 1990-2020: a systematic review and meta-analysis. Lancet Glob Health 2017;5(12):e1221-e34.

[12] Tzekov R. Full-field ERG in diabetic retinopathy: a screening tool? Graefes Arch Clin Exp Ophthalmol 2015;253(7):987-8.

[13] Kempen JH, O'Colmain BJ, Leske MC, et al. The prevalence of diabetic retinopathy among adults in the United States. Arch Ophthalmol 2004;122(4):552-63.

[14] Willis JR, Doan QV, Gleeson M, et al. Vision-related functional burden of diabetic retinopathy across severity levels in the United States. JAMA Ophthalmol 2017;135(9):926-32.

[15] Wang SY, Andrews CA, Herman WH, et al. Incidence and risk factors for developing diabetic retinopathy among youths with type 1 or type 2 diabetes throughout the United States. Ophthalmology 2017;124(4):424-30.
[16] Mathur R, Bhaskaran K, Edwards E, et al. Population trends in the 10-year incidence and prevalence of diabetic retinopathy in the UK: a cohort study in the Clinical Practice Research Datalink 2004-2014. BMJ Open 2017;7(2):e014444.

[17] Medeiros MD, Mesquita E, Papoila AL, et al. First diabetic retinopathy prevalence study in Portugal: RETINODIAB study-evaluation of the screening programme for Lisbon and Tagus Valley region. Br J Ophthalmol 2015;99(10):1328-33.

[18] Hammes HP, Welp R, Kempe HP, et al. Risk factors for retinopathy and DME in type 2 diabetes-results from the German/Austrian DPV Database. PLoS One 2015;10(7):e0132492.

[19] Andersen N, Hjortdal JØ, Schielke KC, et al. The Danish registry of diabetic retinopathy. Clin Epidemiol 2016;8:613-9.

[20] Romero-Aroca P, Navarro-Gil R, Valls-Mateu A, et al. Differences in incidence of diabetic retinopathy between type 1 and 2 diabetes mellitus: a nine-year follow-up study. Br J Ophthalmol 2017;101(10):1346-51.

[21] Maroufizadeh S, Almasi-Hashiani A, Hosseini M, et al. Prevalence of diabetic retinopathy in Iran: a systematic review and Meta-analysis. Int $\mathrm{J}$ Ophthalmol 2017;10(5):782-9.

[22] Zhang G, Chen $\mathrm{H}$, Chen W, et al. Prevalence and risk factors for diabetic retinopathy in China: a multihospital-based cross-sectional study. Br J Ophthalmol 2017;101(12):1591-5.

[23] Huang OS, Tay WT, Ong PG, et al. Prevalence and determinants of undiagnosed diabetic retinopathy and vision-threatening retinopathy in a multiethnic Asian cohort: the Singapore Epidemiology of Eye Diseases (SEED) study. Br J Ophthalmol 2015;99(12):1614-21.

[24] Raman R, Ganesan S, Pal SS, et al. Incidence and progression of diabetic retinopathy in urban India: Sankara nethralaya-diabetic retinopathy epidemiology and molecular genetics study (SN-DREAMS II), Report 1. Ophthalmic Epidemiol 2017;24(5):294-302.

[25] Bastawrous A, Mathenge W, Wing K, et al. The incidence of diabetes mellitus and diabetic retinopathy in a population-based cohort study of people age 50 years and over in Nakuru, Kenya. BMC Endocr Disord 2017;17(1):19.

[26] Chang L, Lee AC, Sue W. Prevalence of diabetic retinopathy at first presentation to the retinal screening service in the greater Wellington region of New Zealand 2006-2015 and implications for models of retinal screening. N Z Med J 2017;130(1450):78-88.

[27] Wong TY, Mwamburi M, Klein R, et al. Rates of progression in diabetic retinopathy during different time periods: a systematic review and meta-analysis. Diabetes Care 2009;32(12):2307-13.

[28] Hammes HP. Optimal treatment of diabetic retinopathy. Ther Adv Endocrinol Metab 2013;4(2):61-71.

[29] Khandekar R. Screening and public health strategies for diabetic retinopathy in the Eastern Mediterranean region. Middle East Afr J Ophthalmol 2012;19(2):178-84.

[30] Rasoulinejad SA, Hajian-Tilaki K, Mehdipour E. Associated factors of diabetic retinopathy in patients that referred to teaching hospitals in Babol. Caspian J Intern Med 2015;6(4):224-8. 
[31] Cardoso CRL, Leite NC, Dib E, et al. Predictors of development and progression of retinopathy in patients with type 2 diabetes: importance of blood pressure parameters. Sci Rep 2017;7(1):4867.
[32] Rasoulinejad SA, Iri HO. Determination of serum lipid profile in patients with diabetic macular oedema that referred to Shahid Beheshti and Ayatollah Rouhani Hospitals, Babol during 2011-2012. Caspian J Intern Med 2015;6(2):77-81. 